\title{
IDH1/IDH2 Mutation Analysis
}

National Cancer Institute

\section{Source}

National Cancer Institute. IDH1/IDH2 Mutation Analysis. NCI Thesaurus. Code C157185.

A procedure used to detect and identify mutations in the IDH1 and IDH2 genes. 